\title{
A role for the CXCL12 receptor, CXCR7, in the pathogenesis of human pulmonary vascular disease
}

\author{
Christine M. Costello*, Brian McCullagh*, Katherine Howell*, Michelle Sands*, \\ John A. Belperio", Michael P. Keane*, Sean Gaine "and Paul McLoughlin*
}

ABSTRACT: Given the critical role that endothelial cell dysfunction plays in the pathogenesis of pulmonary hypertensive diseases, we set out to establish if CXCR7, a receptor for the proangiogenic ligand CXCL12, is expressed in the vasculature of human lung diseases and examine its role in mediating CXCL12-induced responses in primary pulmonary human microvascular endothelial cells.

Receptor and ligand expression was examined in control and explanted human hypertensive lungs, in human plasma and in hypoxic rodent lungs, by ELISA and immunohistochemical studies. Functional in vitro experiments examined the role of CXCR7 in CXCL12-induced lung microvascular endothelial cell proliferation, migration, and wound regeneration and repair.

CXCR7 is elevated in the endothelium of explanted human hypertensive lungs and circulating CXCL12 concentrations are significantly elevated in disease. We demonstrate that alveolar hypoxia similar to that found in lung disease increases CXCR7 expression in the pulmonary endothelium. Furthermore, CXCR7 is the receptor through which endothelial cell regeneration and repair, and proliferation, is mediated, whereas signalling via CXCR4 is essential for chemotactic cell migration.

Our findings demonstrate that CXCR7 has a critical but previously unrecognised role to play in endothelial cell proliferation, suggesting that CXCR7-mediated signalling may be functionally important in pulmonary vascular diseases.

KEYWORDS: CXCR7, CXCR4, CXCL12, hypoxia, pulmonary vasculature

$\mathbf{P}$ ulmonary hypertension $(\mathrm{PH})$ occurs in many forms, which are classified into different categories that reflect the diverse causes and sites of injury associated with disease [1]. Irrespective of the initiating event, the underlying aetiology is characterised by abnormal vascular remodelling, persistent vasoconstriction and increased pulmonary vascular resistance. The pulmonary endothelium is increasingly viewed as being critically involved in abnormal vascular remodelling and vasoconstriction in disease. For example, several rodent studies show that vascular endothelial growth factor receptor blockade, either alone or in combination with hypoxia, culminates in severe $\mathrm{PH}$ and the development of vascular lesions similar to those observed in patients with pulmonary arterial hypertension (PAH) [2-4]. The conventional model of pulmonary endothelial dysfunction in
PAH includes resident endothelial cell damage, loss of barrier integrity due to failure of repair and the development of characteristic vascular lesions in the small pulmonary arterioles $[5,6]$. More recently, a role for circulating endothelial progenitor cells in the pathology of vascular lesions in human PAH has been suggested [7].

In a recent study designed to identify genes selectively regulated in primary human pulmonary microvascular endothelial cells in response to hypoxia, we showed that chemokine (C-X-C motif) receptor 7 (CXCR7) was upregulated in these cells in vitro, and in hypoxic murine lungs in vivo [8]. CXCR7 was recently identified as a receptor for the chemokine ligand, CXCL12, which also signals via a second Gprotein coupled receptor, CXCR4 $[9,10]$. Initial studies established that CXCR7 was an atypical chemokine receptor, with no detectable activation
AFFILIATIONS

*UCD School of Medicine and Medical Science, Conway Institute, 'UCD Dept of Respiratory Medicine, Mater Misericordiae University Hospital, Dublin, Ireland, and \#Division of Pulmonary and Critical Care Medicine, Dept of Medicine, David Geffen School of Medicine at UCLA, Los Angeles, CA, USA.

CORRESPONDENCE

C.M. Costello

UCD School of Medicine and

Medical Science, Conway Institute University College Dublin 4 Ireland

E-mail: christine.costello@ucd.ie

Received:

March 242011

Accepted after revision:

Oct 192011

First published online:

Nov 162011 
of heterotrimeric G-proteins upon ligand binding $[9,10]$. However, several modes of action for CXCR7 have now been described in different cells; developmental studies in zebrafish showed that CXCR7 acts as a scavenger receptor generating CXCL12 gradients along which primordial germ cells migrate [11-13], CXCR7 can act as a co-receptor for CXCR4 in transiently transfected cells [14,15], and CXCR7 can signal independently of CXCR4 via $\beta$-arrestin recruitment [16-18]. Signalling via CXCL12 is now recognised to be important in the development of $\mathrm{PH}$; recent studies show that CXCR4 is expressed on the human endothelium in patients with $\mathrm{PAH}$, and blockade of this receptor in animal models prevents the development of hypoxic PH [7, 19]. However, expression of CXCR7 has not been examined in the human endothelium nor has its role in CXCL12-induced responses in $\mathrm{PH}$ been defined. Therefore, the objectives of the present study were to establish if CXCR7 is expressed in human pulmonary hypertensive diseases and examine its role in mediating pulmonary microvascular endothelial cell responses to CXCL12.

\section{MATERIALS AND METHODS}

Additional details on methods are provided in the online supplementary material.

\section{Human studies}

Immunohistochemistry on explanted lung tissue obtained at the time of lung transplantation from idiopathic PAH (IPAH) patients and patients with usual interstitial pneumonia with pulmonary hypertension (UIP-PH) was carried out as previously described [8]. Histologically normal tissue was obtained from lung tissue resected during cancer surgery at a site remote from the tumour. CXCL12 levels were measured in plasma from nonsmoking controls ( $n=15$; mean age 47.1 yrs, 13 females) with no history of underlying lung disease, and age- and sexmatched IPAH patients ( $n=15$; mean age 49.7 yrs, 12 females), by ELISA (DSA00; R\&D Systems, Minneapolis, MN, USA). All experiments with human material were approved by the ethics committees at the Mater Misericordiae University Hospital, Dublin, Ireland and the David Geffen School of Medicine at UCLA, Los Angeles, CA, USA.

\section{Animal studies}

All animal experiments were approved by the animal research ethics subcommittee of University College Dublin, Dublin, Ireland and carried out under license from the Department of Health. Chronic hypoxia was induced by housing adult male C57BL/ 6 mice or Sprague-Dawley rats in 10\% inspired oxygen for 2 days or 3 weeks, respectively, while control animals were housed in normoxic conditions (inspired oxygen 21\%) in the same room. Processing of lung samples for ELISA (MCX120; R\&D Systems) and immunohistochemical analysis is described in the online supplement. The extent of protein staining was determined by quantitative stereological techniques, as previously described [8].

\section{Cell culture studies}

The separate role of CXCR7 and CXCR4 in modulating endothelial wound healing and repair (scratch assay), proliferation (cell count) and migratory (modified Boyden chamber) responses of primary human lung microvascular endothelial cells to CXCL12 was investigated as outlined in the online supplement. Cell experiments were carried out six independent times using concentrations of specific inhibitors of CXCR7 (CCX771 and CCX773; ChemoCentryx, Mountain View, CA, USA) and CXCR4 (AMD3100; Sigma-Aldrich, St Louis, MO, USA) previously shown to effectively block these receptors [17].

\section{Statistical analyses}

Statistical analyses were performed with SPSS 12.0 statistics software. For normally distributed data, values are reported as mean \pm SEM. Comparison of means in two group experiments was carried out using a paired t-test. For non-normally distributed data, statistical comparisons were made using Mann-Whitney U-test. Corrections of multiple comparisons of means were carried out using the Holm-Sidak step-down post hoc test. Values of $\mathrm{p}<0.05$ were considered to be statistically significant.

\section{RESULTS \\ CXCR7 is highly expressed in human pulmonary hypertensive diseases compared to control lungs}

We found that CXCR7 expression was low or frequently absent in the vasculature of control subjects (fig. 1a) but was widespread in the lungs of IPAH patients, with strong positive staining observed in the thickened intimal layer of remodelled blood vessels (fig. 1b). CXCR4 and CXCL12 were also more highly expressed in the microvasculature of IPAH (fig. 1e and $1 \mathrm{~h}$, respectively) compared to histologically normal lungs (fig. $1 \mathrm{~d}$ and $1 \mathrm{~g}$, respectively). We also show for the first time that infiltrating cells, clearly observed in the alveolar spaces of IPAH lungs, were stained for CXCR7 (fig. 2b), whereas only faint CXCR7 staining was observed in these cells in normal lungs (fig. 2a). This marked increase in protein expression in infiltrating cells in IPAH was also observed with CXCR4 (fig. 2e) compared to control tissue (fig. 2d). Likewise, CXCL12 expression was also increased in infiltrating cells in pulmonary hypertensive lungs (fig. 2h) compared to histologically normal lungs (fig. 2g). Lungs from a second pulmonary hypertensive patient group, UIP-PH, also showed marked staining of all three proteins in the endothelium of blood vessels and infiltrating inflammatory cells (representative images are shown in supplementary figures S1 and S2). No positive staining was observed with appropriate irrelevant immunoglobulin $(\mathrm{Ig}) \mathrm{G}$ isotype controls for the three proteins of interest (figs 1 and 2; S1 and S2; panels $\mathrm{c}, \mathrm{f}$ and i).

\section{CXCL12 is significantly elevated in patients with IPAH}

ELISA analysis of CXCL12 concentrations in plasma from IPAH patients indicated that CXCL12 concentrations were significantly elevated in the patient group $(n=15)$ when compared to non-diseased age- and sex-matched control subjects $(n=15)$ (fig. 3$)$.

\section{CXCR7, CXCR4 and CXCL12 are elevated in hypoxic rodent lungs}

Immunohistochemical staining showed that CXCR7 protein was expressed basally in the normoxic lung (fig. 4a) and increased substantially after 2 days in hypoxia $\left(\begin{array}{lll}10 \% & \mathrm{O}_{2}\end{array}\right)$ (fig. 4b). CXCR7 immunoreactivity was clearly observed in the alveoli walls and in the vessel endothelium. Quantification of the volume of CXCR7-stained tissue per unit volume of alveolar wall in the lung showed that CXCR7 protein was 

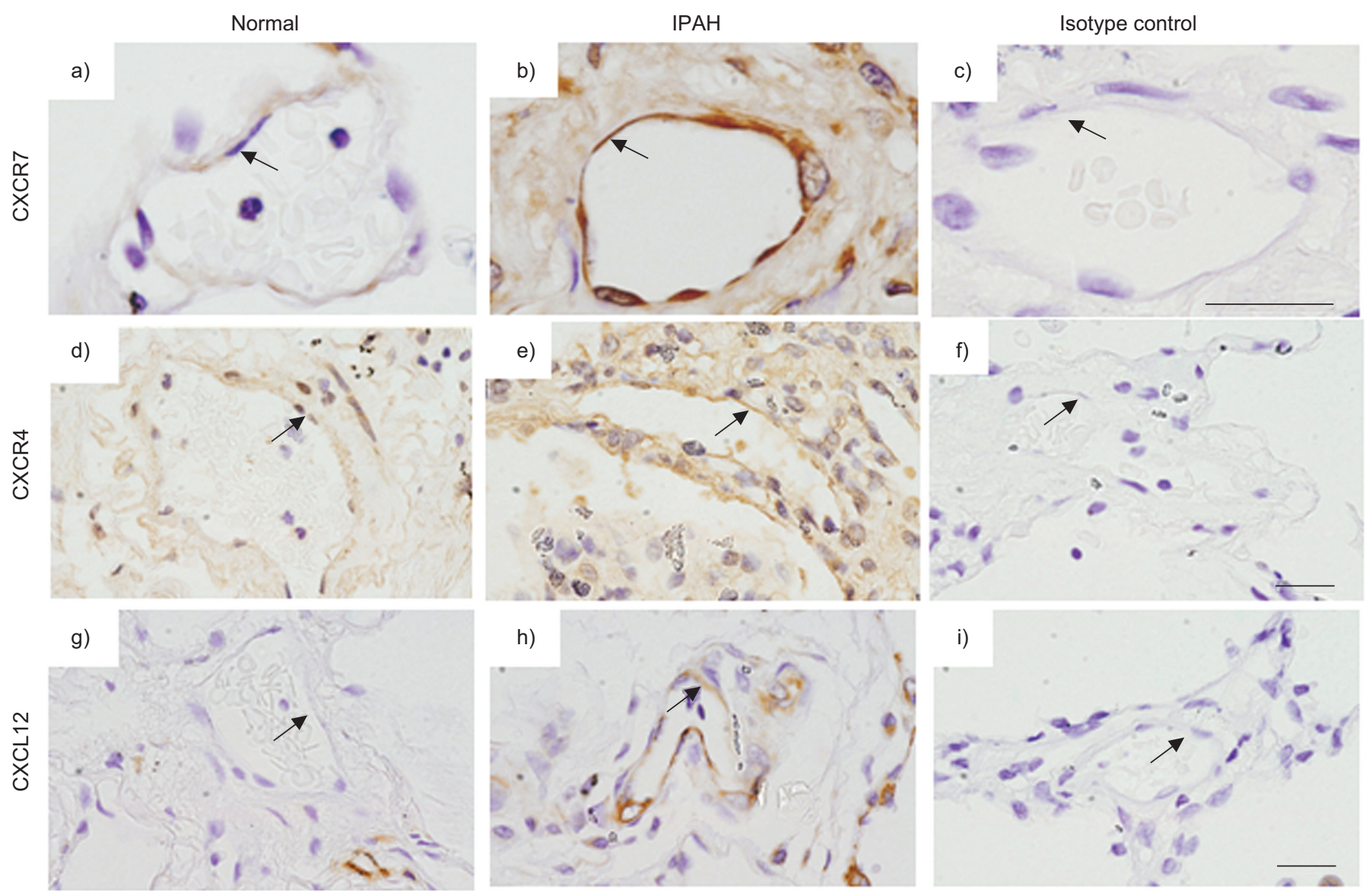

FIGURE 1. Immunostaining in the vasculature of control and idiopathic pulmonary arterial hypertension (IPAH) patient lungs. CXCR7 (a and b), CXCR4 (d and e) and CXCL12 ( $g$ and $\mathrm{h}$ ) protein is expressed (brown colour) in the endothelium of the pulmonary vasculature of control lungs and in IPAH. Staining was absent when isotypematched antibodies were tested on the tissue sections (immunoglobulin (Ig) $G_{2 a}$ for $C X C R 7$ (c), IgG $G_{2 b}$ for $C X C R 4$ (f), IgG for CXCL12 (i). Representative images from three control and three IPAH patients are shown. Arrows indicate endothelium of vessels. Scale bars $=20 \mu \mathrm{m}(\times 100$ objective $)$.

significantly higher in hypoxic lungs than in normoxic lungs (fig. 4d). Similarly, basal CXCR4 expression observed in normoxic murine lungs (fig. 4e) increased significantly with exposure to hypoxia (fig. $4 \mathrm{f}$ ), quantification of protein staining as described above showed that CXCR4 protein was also significantly higher in hypoxia when compared to normoxia (fig. 4h). The specificity of CXCR7 and CXCR4 staining was confirmed with appropriate irrelevant IgG controls (fig. 4c and g).

We next examined CXCL12 concentrations and protein localisation in hypoxic murine lungs. ELISA analysis indicated that CXCL12 concentration in whole lung homogenates from hypoxic mice was significantly elevated when compared to normoxic lungs (fig. 5a). In a separate cohort of mice, immunohistochemical staining with anti-CXCL12 antibody showed low levels of CXCL12 staining in normoxic endothelial cells (fig. 5b). In contrast, the endothelium of the lung microvasculature was highly positive for CXCL12 when exposed to hypoxia (fig. 5c). The specificity of positive staining was established with an irrelevant rabbit polyclonal IgG (fig. 5d).

It is well documented that rats develop marked pulmonary vascular remodelling, similar to humans and other species, after chronic hypoxia exposure, in contrast to mice, in which relatively minor remodelling is observed even though they develop sustained pulmonary hypertension [20]. Thus we also examined receptor expression in a rat model of hypoxic pulmonary hypertension (Sprague-Dawley rats exposed to $10 \% \mathrm{O}_{2}$ for 3 weeks). Immunohistochemical analysis showed low levels of CXCR7 protein in normoxic lung (fig. S3a in the online supplementary material) whereas strong CXCR7 staining was observed in hypoxic lung tissue, particularly in the vascular endothelium (fig. S3b). Stereological quantification of CXCR7 immunoreactivity confirmed that the extent of CXCR7 protein expression was significantly higher in hypoxia (fig. S3d). Likewise, basal CXCR4 expression observed in normoxic murine lungs (fig. S3e) increased significantly with exposure to hypoxia (fig. S3f). Stereological quantification of the extent of protein staining showed that CXCR4 was also significantly higher in hypoxia when compared to normoxia (fig. S3h). The specificity of positive immunohistochemical staining was confirmed with appropriate irrelevant IgG controls (figs S3c and g).

\section{Inhibition of CXCR7 blocks CXCL12 induced monolayer regeneration and repair}

In order to establish the potential roles of CXCR7 and CXCR4 in mediating CXCL12-induced responses in primary human lung microvascular endothelial cells, we first confirmed that 

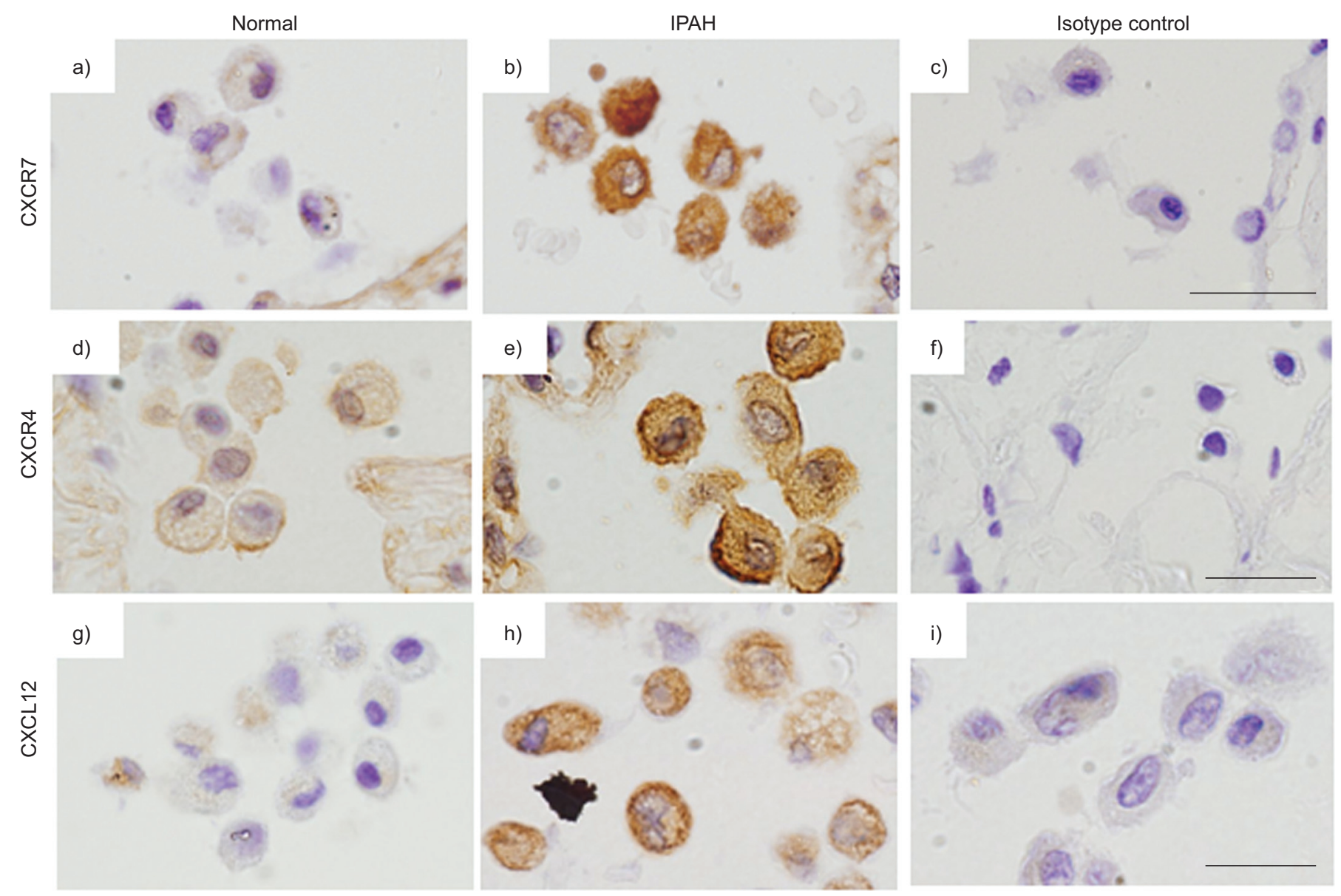

FIGURE 2. Immunostaining of infiltrating inflammatory cells in control and idiopathic pulmonary arterial hypertension (IPAH) patient lungs. Representative images of infiltrating cells in the lung in normal controls showed that basal positive CXCR7 staining (a) increased in IPAH patients (b). A similar pattern of increased positive staining in disease was observed with CXCR4 (control (d) versus IPAH (e)) and CXCL12 (control (g) versus IPAH (h)). No staining was observed in these cells with appropriate isotype controls (immunoglobulin (Ig) $\mathrm{G}_{2 A}$ for $\mathrm{CXCR7}$ (c), IgG $\mathrm{G}_{2 B}$ for CXCR4 (f), $\lg \mathrm{G}_{1}$ for $\mathrm{CXCL12}$ (i)). Representative images from three control and three IPAH patients are shown. Scale bars $=20 \mu \mathrm{m}(\times 100$ objective $)$.

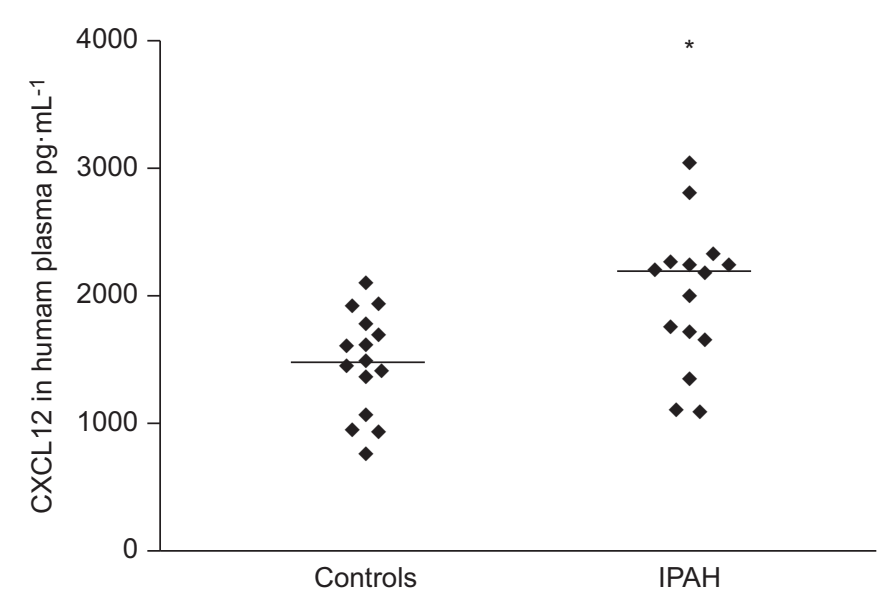

FIGURE 3. CXCL12 determination by ELISA in human plasma. CXCL12 levels in plasma samples from controls $(n=15)$ and idiopathic pulmonary arterial hypertensive patients $(n=15)$. Protein levels of this potent pro-angiogenic cytokine were significantly increased in the diseased population. *: $p<0.05$. Horizontal bars indicate median values. both receptors were expressed by these cells (fig. 6a). We next examined the effect of receptor inhibition on the regeneration and repair of endothelial cell monolayers; preliminary concentration-response experiments using our in vitro wound healing model showed that recombinant CXCL12 (10 nM) elicited maximum wound healing (data not shown). We next determined if a small molecule inhibitor of CXCR7, namely CCX771, altered the CXCL12 induced wound healing response. As shown in figure $6 \mathrm{~b}$, dose-response experiments with CCX771 demonstrated that maximal inhibition of wound healing was observed with $1 \mu \mathrm{M}$ of CCX771. A second CXCR7 inhibitor, CCX773, also inhibited CXCL12-induced wound healing (fig. S4a in the online supplementary material). In contrast, increasing concentrations of the CXCR4 receptor antagonist, AMD3100 (0.01-1 $\mu \mathrm{M})$, had no effect on CXCL12induced wound healing (fig. 6c).

\section{Inhibition of CXCR7 blocks proliferation but not migration of human pulmonary microvascular endothelial cells in vitro}

To further examine the wound healing response, we next established that CXCL12 $(10 \mathrm{nM})$ induces proliferation of microvascular lung endothelial cells, a proliferative response 

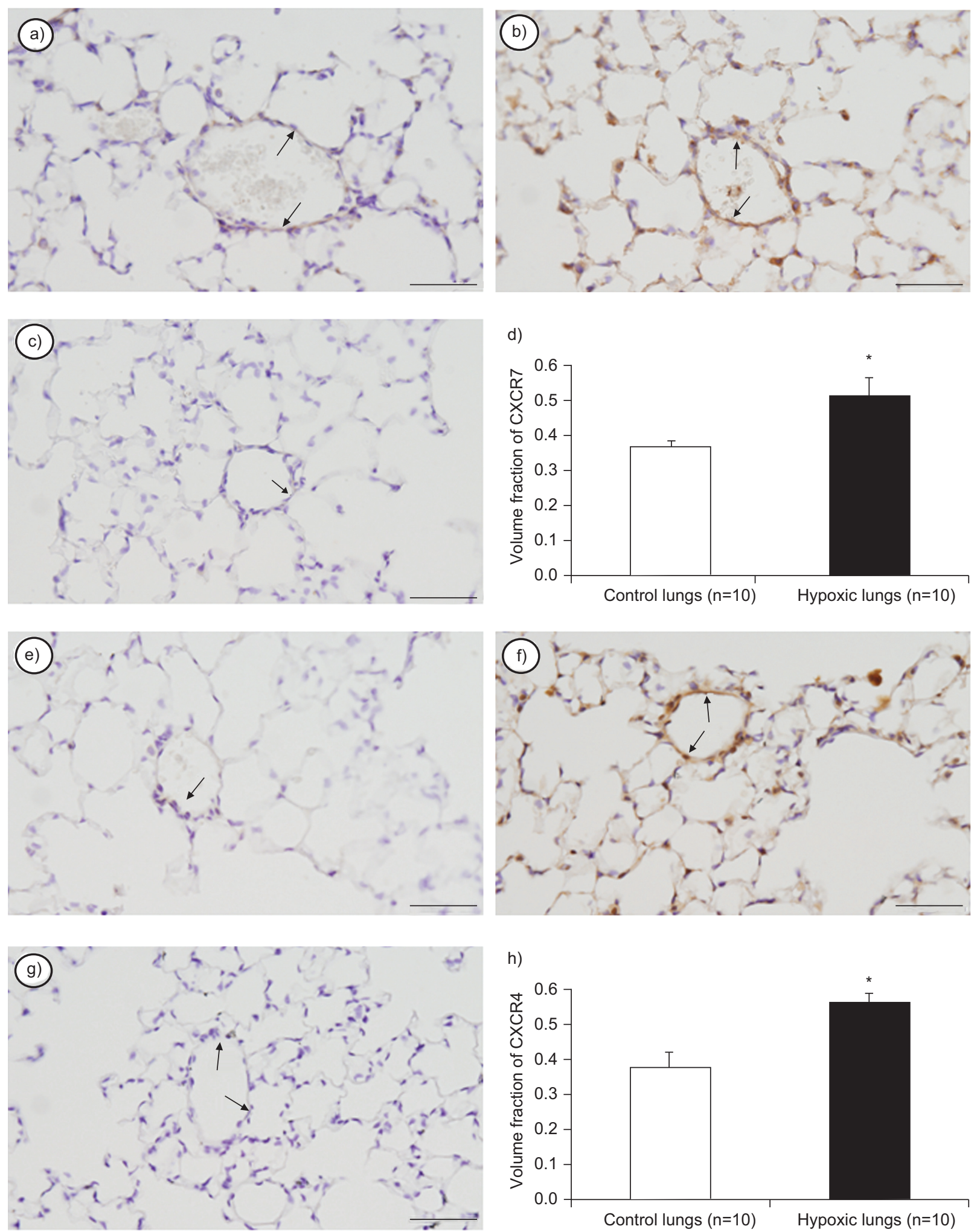

FIGURE 4. Immunohistochemical localisation of CXCR7 and CXCR4 in normoxic and hypoxic murine lungs. Immunohistochemical staining (brown colour) indicated that CXCR7 protein was expressed basally in normoxic lung tissue (a) and increased with exposure to hypoxia (b). Using stereology, the volume of cells within the alveolar wall that were CXCR7-positive was expressed as a fraction of total alveolar wall volume (volume fraction) and was significantly higher in hypoxic lungs (d). Similarly, the volume fraction of CXCR4-stained tissue in normoxic (e) and hypoxic ( $f$ ) lungs showed that CXCR4 protein was significantly higher in the hypoxic lungs (h). No staining was observed with irrelevant rabbit polyclonal $\operatorname{lgG}(\mathrm{c}$ and $\mathrm{g})$. $^{*} \mathrm{p}<0.05$ 


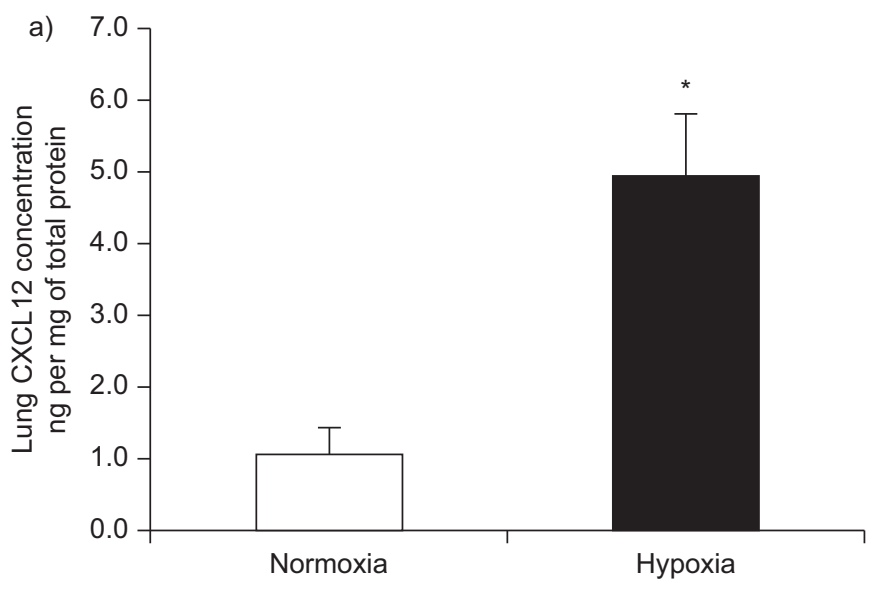

FIGURE 5. CXCL12 quantification and localisation in murine lungs. a) $C X C L 12$ protein was significantly higher in hypoxic lung homogenates $(n=10)$ compared to normoxia $(n=9)$ by ELISA. *: $p<0.05$. Immunohistochemical staining indicated that a low basal level of CXCL12 was observed in the endothelium of normoxic blood vessels (b) in the gas exchange region, which increased with exposure to hypoxia (c). No staining was observed with irrelevant rabbit polyclonal $\lg G$ (d). Arrows indicate vessels. Scale bars $=50 \mu \mathrm{m}(\times 40$ objective $)$.
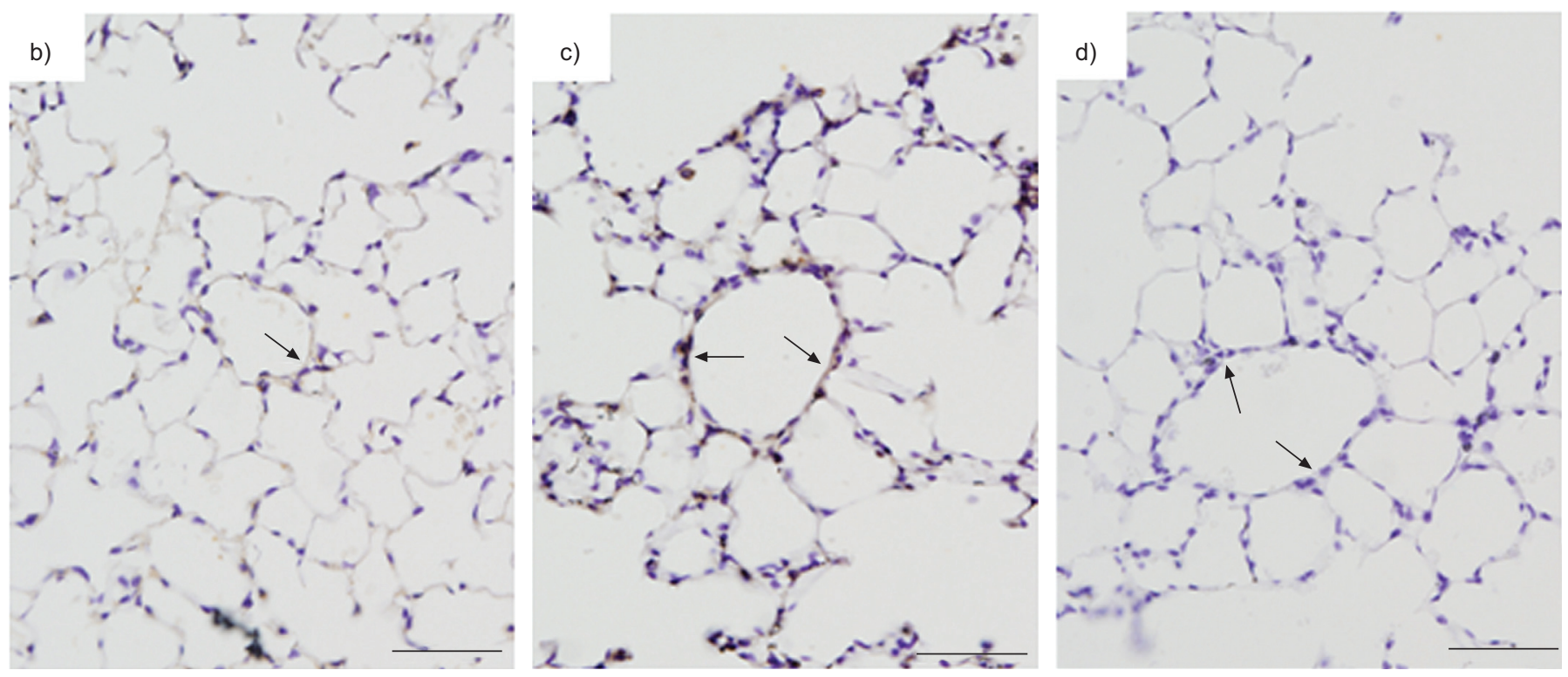

that was inhibited by CCX771 $(1 \mu \mathrm{M})$ (fig. 7a). The second CXCR7 inhibitor (CCX773; $1 \mu \mathrm{M})$ was also capable of inhibiting cell proliferation (fig. S4b in the online supplementary material). In contrast, inhibition of CXCR4 with the CXCR4 receptor antagonist AMD3100 $(1 \mu \mathrm{M})$ had no effect on microvascular lung endothelial cell proliferation (fig. $7 \mathrm{~b}$ ). These results suggest that CXCL12 enhances proliferation of endothelial cells through interaction with CXCR7, whereas CXCR4 is not required in this process.

\section{Inhibition of CXCR7 has no effect on migration of human pulmonary microvascular endothelial cells in vitro}

In a Boyden chamber assay, cell migration towards vehicle alone was low (fig. 8a), which was in marked contrast to the chemotactic response observed in the presence of CXCL12 (10 nM) (fig. 8b). Subsequent experiments showed that the CXCL12-induced increase in microvascular lung endothelial cell migration was unaffected by CXCR7 inhibition (CCX771; $1 \mu \mathrm{M}$ ) (fig. 8c), a similar effect was observed with the second CXCR7 inhibitor, CCX773 (fig. S4c in the online supplementary material). In contrast, endothelial cell migration was significantly inhibited by CXCR4 blockade (AMD3100, $1 \mu \mathrm{M}$ ) (fig. 8d). These results suggest that, of the two CXCL12 receptors, CXCR4 is primarily required for the chemotactic response of endothelial cells to CXCL12.

\section{DISCUSSION}

Given the critical role that endothelial cell dysfunction plays in the pathogenesis of pulmonary hypertensive diseases, we set out to establish if CXCR7, a receptor for the potent proangiogenic ligand, CXCL12, was expressed in human pulmonary hypertensive diseases (IPAH and UIP-PH) and in the setting of hypoxia-induced $\mathrm{PH}$. We show for the first time that CXCR7 is highly expressed in the pulmonary vascular endothelium of explanted human hypertensive lungs and circulating concentrations of its ligand are significantly elevated in the plasma of IPAH patients. Our study is the first demonstration that hypoxia increases CXCR7 expression in the pulmonary endothelium in vivo. Furthermore, CXCR7 has a distinct role to play in the CXCL12-induced responses of primary pulmonary human endothelial cells from that mediated by CXCR4; i.e. our data suggest that CXCR7 is the receptor through which endothelial cell proliferation, and regeneration and repair of endothelial cell monolayers, is mediated, whereas signalling via CXCR4 is essential for chemotactic cell migration. These results, taken together with 
a)

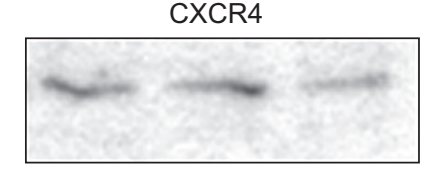

b) 80

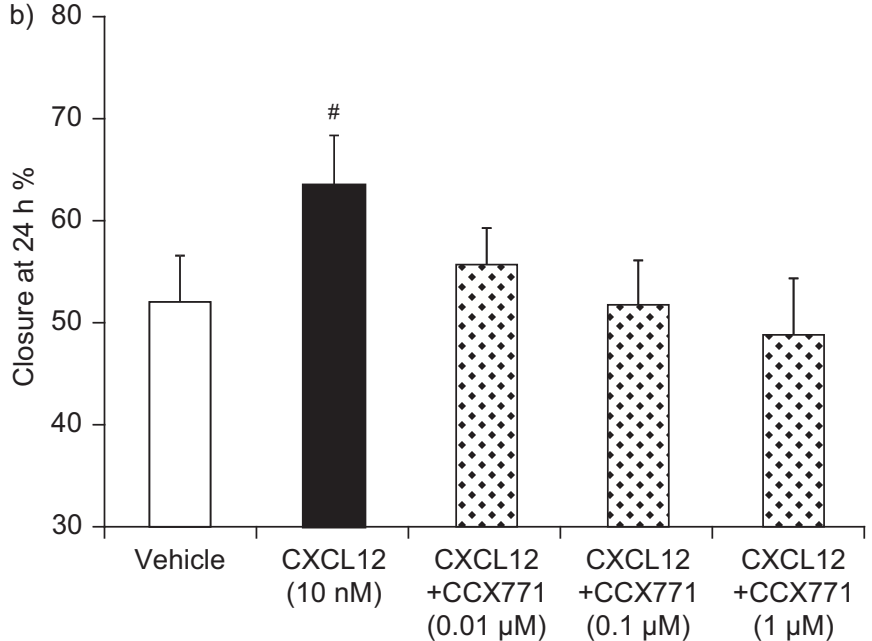

CXCR7

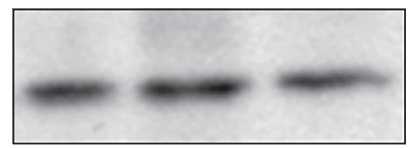

$(1 \mu \mathrm{M})$

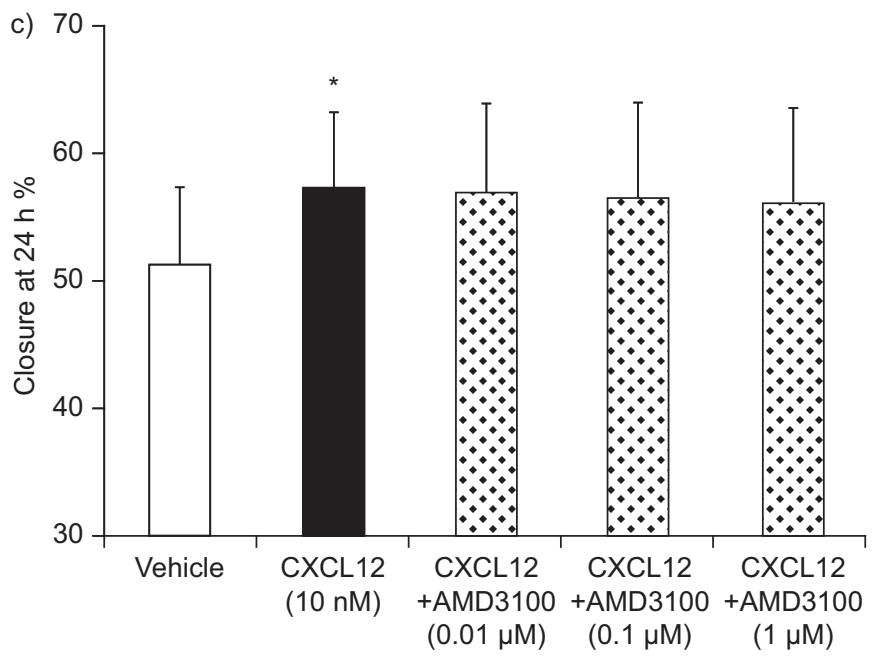

FIGURE 6. CXCL12-induced wound healing of human pulmonary microvascular endothelial cells was blocked by CXCR7 inhibition. a) Western blot analysis confirmed that CXCR4 and CXCR7 protein were expressed by human pulmonary microvascular endothelial cells. Cell pellets from three independent experiments are shown. b) The specific CXCR7 inhibitor, CCX771, inhibited CXCL12 (10 nM)induced primary human pulmonary microvascular endothelial cell wound healing. C) The CXCR4 inhibitor, AMD3100, had no effect on wound healing in the same concentration range $(0.01-1.0 \mu \mathrm{M})$. All experiments were carried out six independent times. *: significant difference $(\mathrm{p}<0.05)$ between control and CXCL12; ${ }^{*}$ : significant difference $(p<0.05)$ from all other groups.

marked staining of CXCR7 in vascular lesions in human lung disease, suggest CXCR7-mediated signalling may be functionally important in pulmonary hypertensive disease.

In a previous microarray study, we originally showed that CXCR7 mRNA was selectively upregulated in the hypoxic murine lung in vivo while remaining unchanged in systemic organs [8]. In humans, CXCR7 was previously reported not to
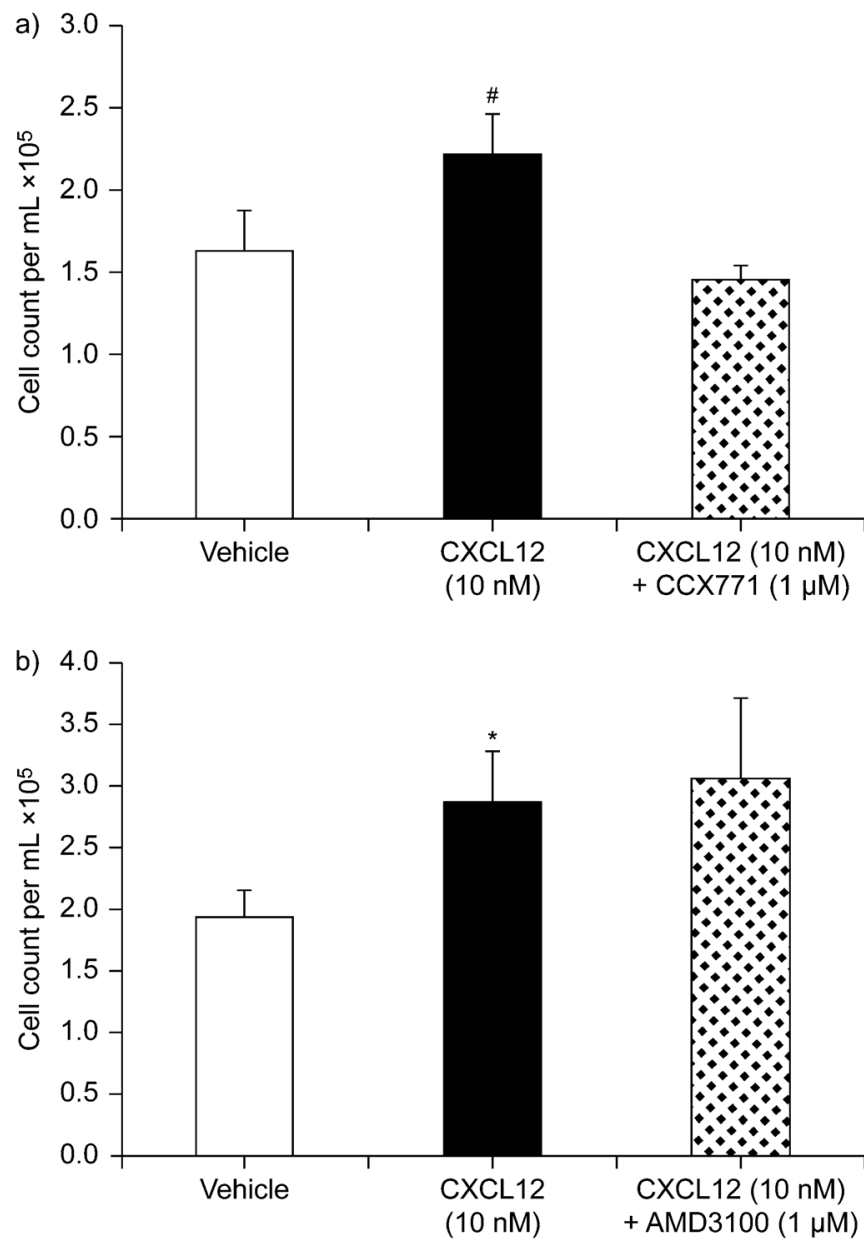

FIGURE 7. CXCR7 inhibition blocked CXCL12-induced proliferation of human pulmonary microvascular endothelial cells. a) CXCL12 (10 nM)-induced primary human pulmonary microvascular endothelial cell proliferation was blocked by CXCR7 inhibition (CCX771; $1.0 \mu \mathrm{M}$ ), whereas b) CXCR4 blockade (AMD3100; $1.0 \mu \mathrm{M})$ did not significantly alter the CXCL12-induced proliferative effect. All experiments were carried out six independent times. *: significant difference $(p<0.05)$ between control and CXCL12; \#: significant difference $(\mathrm{p}<0.05)$ from all other groups.

be expressed in endothelium of normal systemic organs in vivo but highly expressed in tumour-associated vasculature or in endothelial cells during human renal allograft rejection [21, 22]. We now show that, contrary to the vasculature of systemic organs, CXCR7 is present on the pulmonary endothelium under normal circumstances in vivo. Furthermore, alveolar hypoxia similar to that found in lung disease results in increased CXCR7 expression by the pulmonary microvascular endothelium. In support of the contention that CXCR7 expression is different in the pulmonary circulation from that in the systemic circulation, we also show that CXCR7 is expressed by normal lung endothelial cells in vitro, whereas previous reports suggest that CXCR7 is only expressed by endothelial cells that are first activated by pro-inflammatory cytokines (i.e. tumour necrosis factor- $\alpha$ and interleukin-1 $\beta$ ) or in tumour or transformed cell lines [10, 17].

We show for the first time that CXCR7 is highly expressed in the grossly remodelled microvasculature of human hypertensive 

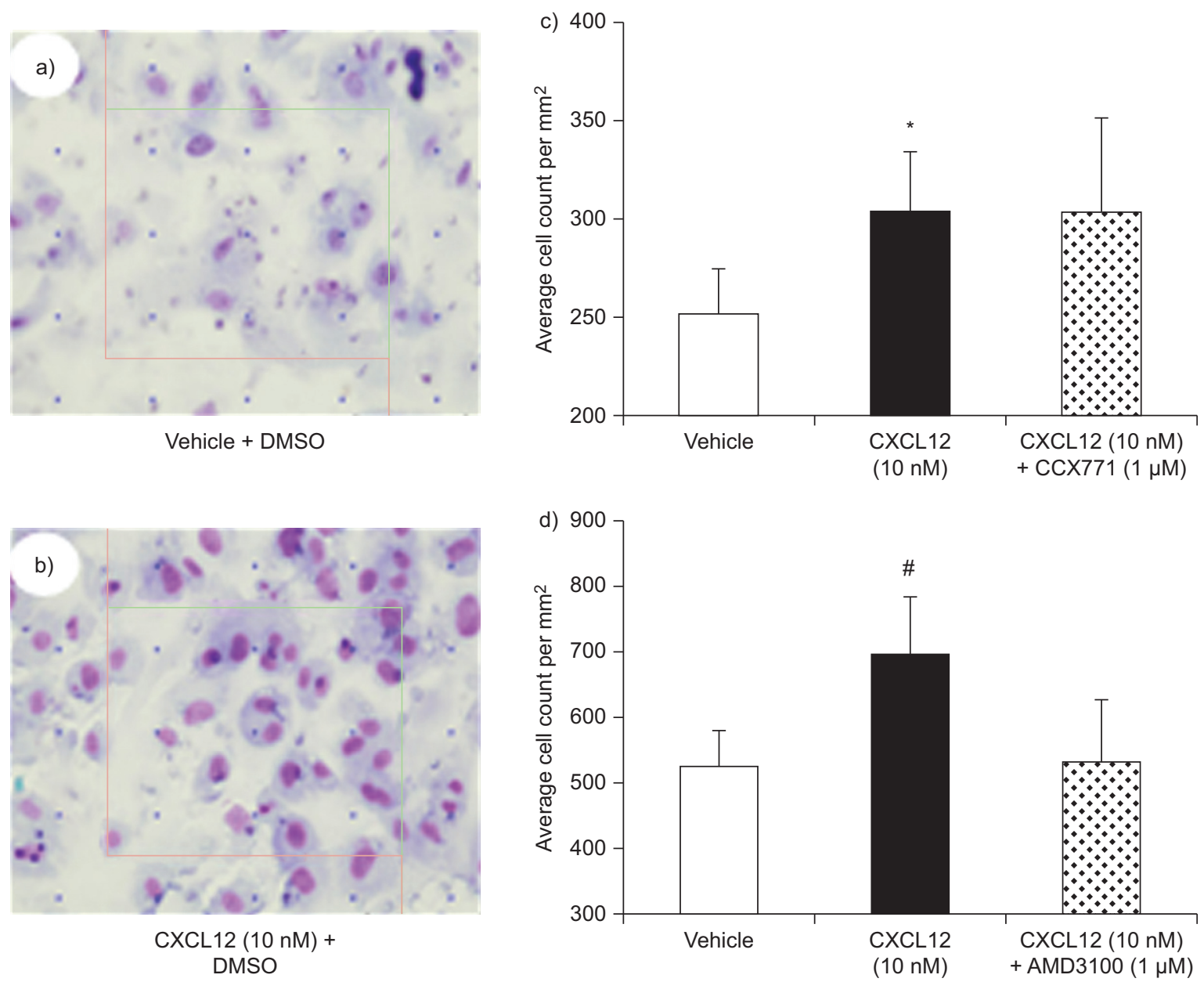

FIGURE 8. CXCR4 inhibition blocked CXCL12-induced migration of human pulmonary microvascular endothelial cells. Representative images of pulmonary endothelial cell migration with cells treated with vehicle alone (a) or with CXCL12 (10 nM) (b) are shown. c) CXCL12 (10 nM) significantly induced cell migration, an effect that was unaltered by inhibition of CXCR7 (CCX771; $1 \mu \mathrm{M})$. d) In contrast, cell migration was blocked by CXCR4 inhibition (AMD3100; $1 \mu \mathrm{M}$ ). All experiments were carried out six independent times. *: significant difference $(p<0.05)$ between control and CXCL12; ${ }^{*}$ : significant difference $(p<0.05)$ from all other groups.

diseases, such as IPAH and UIP-PH, whereas CXCR7 expression is low or frequently absent in the vasculature of control subjects, suggesting that this pathway may be involved in disease pathogenesis. We also observed that CXCR4 and CXCL12 were more highly expressed in remodelled vessels in both hypertensive diseases compared to controls, supporting a recent report that these two latter proteins were expressed in concentric and plexiform lesions characteristically seen in IPAH [7]. Since the initial description of CXCR7 as a second receptor for CXCL12, several studies have established an important physiological function of CXCR7 in vascular development and disease [10, 23]. For example, morpholinomediated knockdown of CXCR7 in zebrafish results in disrupted vascular formation [21]. Furthermore, the majority of CXCR7-/- mice, or mice conditionally deficient for CXCR7 in the endothelium, die at birth due to ventricular septum and heart valve malformation; in wild-type animals, CXCR7 is expressed in the microvasculature of these sites [15]. Both these studies suggest a critical role for CXCR7 in normal vascular homeostasis during development. We now show that CXCL12 and both receptors are expressed by the lung endothelium, and that circulating CXCL12 concentrations are significantly elevated in IPAH; this suggests that autocrine/paracrine modes of actions may be at play in the setting of lung disease. In contrast to our finding, MONTANI et al. [24] showed that circulating levels of CXCL12 were not significantly elevated in IPAH. The cause of this discrepancy is not immediately apparent but differences in patient populations between the two studies may be a factor. For example, a recent paper demonstrated that CXCL12 concentrations are age dependent [25]; in our study we closely agematched our study populations so this was not a factor in significantly elevated CXCL12 measured in our patients. Additional studies in larger IPAH cohorts and other hypertensive patient groups are now warranted but, nevertheless, our finding of elevated CXCL12 in IPAH plasma, together with elevated expression of both the ligand and its receptors in explanted human hypertensive lungs, are compatible with an important role for this biological axis in PAH.

In addition to demonstrating that CXCR7 is elevated in the grossly remodelled microvasculature of human hypertensive diseases, we now demonstrate that CXCR7 is highly expressed on infiltrating cells in diseased lungs, as was CXCR4. We have 
yet to elucidate the exact consequence of receptor expression on these cells in IPAH and UIP-PH; however, CXCL12 is a potent chemoattractant of T-lymphocytes and monocytes in vitro and CXCL12 upregulation has been shown to precede accumulation of mononuclear cells in vessel walls in a murine model of hypoxic PH in vivo [26, 27]. Once in the lung, these cells are purported to contribute to disease pathogenesis primarily via the local release of cytokines and growth factors which modulate changes in resident endothelial, smooth muscle or adventitial cells. Indeed, elevated levels of circulating pro-inflammatory cytokines and inflammatory infiltrates in vascular lesions in PAH are now well described [5, 6, 28].

The exact role of CXCR7 signalling in hypoxia-induced lung disease in vivo is as yet unclear. The fundamental principle that CXCL12 is important in hypoxic PH is well established by animal studies showing that a reduction in CXCL12 signalling via CXCR4 alleviated hypoxia-induced $\mathrm{PH}$ in mice $[19,29,30]$. A recent study also suggested that CXCR7 blockade may reduce hypoxia-induced perivascular accumulation of c-kit+ haematopoietic cells in remodelled vessels [19]. Our in vivo immunohistochemical studies show that the extent of CXCR7 protein is significantly elevated in the alveolar walls and in the vascular endothelium of small pulmonary vessels in both the hypoxic murine lung $\left(10 \% \mathrm{O}_{2}\right.$ for 2 days) and in a rat model of hypoxic $\mathrm{PH}\left(10 \% \mathrm{O}_{2}\right.$ for 3 weeks), but further studies are required to establish the precise role of CXCR7 signalling in hypoxia-induced disease pathogenesis.

In the present study, we demonstrate a functional outcome of CXCR7 signalling in lung endothelial cells. It is now well established that CXCR7 (unlike CXCR4) does not couple G proteins, with several studies reporting the absence of GTP hydrolysis, calcium mobilisation and chemotaxis upon ligand binding to CXCR7 [10, 14]. In fact, the CXCR7-mediated cellular responses to CXC12 are not yet fully elucidated, but it has been shown that ligand binding triggers $\beta$-arrestin-2 association with CXCR7 and ligand/receptor internalisation in vitro [16-18]. CXCR7 expression on the endothelium is involved in various biological processes, such as transendothelial migration of tumour cells and human renal progenitors in vitro $[17,31]$ and primordial germ cell migration during zebrafish development in vivo [11-13]. Furthermore, CXCR7 signalling has also been shown to promote tumour growth in vivo $[10,21,32]$ and exerts pro-survival and anti-apoptotic effects in vitro [10,31]. Our experiments now show a further important action of CXCR7 in pulmonary endothelial cells, namely a role in cell proliferation. In our in vitro cell experiments, blockade of CXCR7 significantly reduced the ability of CXCL12-induced pulmonary endothelial cells to proliferate, and repair and regenerate, a wounded endothelial cell monolayer, whereas CXCL12-induced pulmonary vascular endothelial cell migration was unaffected by CXCR7 inhibition, but significantly inhibited by CXCR4 blockade. Distinct roles for receptor mediated signalling have previously been reported in the homing of human renal progenitor cells where CXCL12-induced migration was blocked by CXCR4 inhibition, whereas cell survival was blocked by CXCR7 inhibition [31]. Interestingly, although CXCL12-CXCR4 interaction was responsible for renal progenitor cell recruitment, both receptors were required for transendothelial migration underlining a complex interplay between CXCR7 and CXCR4 signalling
[31]. Since coordinated proliferation and migration is required for normal endothelial repair and angiogenesis, alterations in the normal balance of these receptors could be important in the pathogenesis of $\mathrm{PH}$, given that dysregulated endothelial cell proliferation resulting in intimal hyperplasia is a pathological hallmark of hypertensive lung disease $[5,6]$.

It is interesting that CXCR4 inhibition blocked CXCL12 stimulated migration of endothelial cells in the Boyden chamber experiment but had no effect on wound repair in the scratch assay. However, there is a growing understanding that chemotaxis (directed individual cell motility along a chemical gradient, as measured in Boyden chamber experiments) and chemokinesis (non-directional motility of cells due to the presence of a chemical substance, as occurs during coordinated wound closure by the cell monolayer) represent two distinct forms of migration. For example, the coordinated epidermal growth factor (EGF)-stimulated wound closure of mouse colonic epithelial cell monolayers requires phosphatidylinositol 3-kinase, protein kinase $C$, and JNK/SAPK activity, whereas chemotaxis (Boyden chamber) to EGF did not [33]. These findings indicate that the specific signalling pathways necessary for chemotaxis are different from those required for chemokinesis during wound healing. Our findings now provide evidence of separate pathways regulating CXCL12-induced chemotaxis and the process of chemokinesis required for pulmonary microvascular endothelial wound healing.

In summary, we show that the predominant cells expressing CXCR7 in the lung in vivo are endothelial cells and that CXCL12 is elevated in the plasma of human IPAH. We also show that CXCL12 induces proliferation, migration and wound healing of human microvascular endothelial cells in vitro. CXCR7 and CXCR4 appear to have distinct roles in these latter processes; in particular, we report a previously unrecognised function of CXCR7 in endothelial cell proliferation and wound repair, suggesting that this protein may have an important role to play in the vascular lesions observed in disease. We propose that signalling via CXCR7 plays a key role in pulmonary endothelial cell responses to CXCL12 in disease, suggesting that therapeutic manipulation of this pathway may present novel treatment opportunities.

\section{SUPPORT STATEMENT}

This work was funded by grants from the Health Research Board and the Higher Education Authority (Programme for Research in ThirdLevel Institutions) and an unrestricted research grant from Actelion. The funding bodies had no role in study design, data collection and analysis, decision to publish or preparation of the manuscript.

\section{STATEMENT OF INTEREST}

Statements of interest for B. McCullagh, M.P. Keane and S. Gaine, and for the study itself, can be found at www.erj.ersjournals.com/site/ misc/statements.xhtml

\section{ACKNOWLEDGEMENTS}

The technical assistance of S. Hewage and R. Hines (UCD School of Medicine and Medical Science, Conway Institute, Dublin, Ireland) is gratefully acknowledged. CXCR7 inhibitors were kindly provided by M.E. Penfold at ChemoCentryx Inc., Mountain View, CA, USA. The authors thank the patients and staff at the participating centres in the study. 


\section{REFERENCES}

1 Simonneau G, Galie N, Rubin LJ, et al. Clinical classification of pulmonary hypertension. J Am Coll Cardiol 2004; 43: 5S-12S.

2 Taraseviciene-Stewart L, Kasahara Y, Alger L, et al. Inhibition of the VEGF receptor 2 combined with chronic hypoxia causes cell death-dependent pulmonary endothelial cell proliferation and severe pulmonary hypertension. FASEB J 2001; 15: 427-438.

3 Voelkel NF, Cool C, Taraceviene-Stewart L, et al. Janus face of vascular endothelial growth factor: the obligatory survival factor for lung vascular endothelium controls precapillary artery remodeling in severe pulmonary hypertension. Crit Care Med 2002; 30: S251-S256.

4 Le Cras TD, Markham NE, Tuder RM, et al. Treatment of newborn rats with a VEGF receptor inhibitor causes pulmonary hypertension and abnormal lung structure. Am J Physiol Lung Cell Mol Physiol 2002; 283: L555-L562.

5 Rabinovitch M. Molecular pathogenesis of pulmonary arterial hypertension. J Clin Invest 2008; 118: 2372-2379.

6 Humbert M, Montani D, Perros F, et al. Endothelial cell dysfunction and cross talk between endothelium and smooth muscle cells in pulmonary arterial hypertension. Vascul Pharmacol 2008; 49: 113-118.

7 Toshner M, Voswinckel R, Southwood M, et al. Evidence of dysfunction of endothelial progenitors in pulmonary arterial hypertension. Am J Respir Crit Care Med 2009; 180: 780-787.

8 Costello CM, HowellK, Cahill E, et al. Lung-selective gene responses to alveolar hypoxia: potential role for the bone morphogenetic antagonist gremlin in pulmonary hypertension. Am J Physiol Lung Cell Mol Physiol 2008; 295: L272-L284.

9 Balabanian K, Lagane B, Infantino S, et al. The chemokine SDF-1/ CXCL12 binds to and signals through the orphan receptor RDC1 in T lymphocytes. J Biol Chem 2005; 280: 35760-35766.

10 Burns JM, Summers BC, Wang Y, et al. A novel chemokine receptor for SDF-1 and I-TAC involved in cell survival, cell adhesion, and tumor development. J Exp Med 2006; 203: 2201-2213.

11 Boldajipour B, Mahabaleshwar H, Kardash E, et al. Control of chemokine-guided cell migration by ligand sequestration. Cell 2008; 132: 463-473.

12 Dambly-Chaudiere C, Cubedo N, Ghysen A. Control of cell migration in the development of the posterior lateral line: antagonistic interactions between the chemokine receptors CXCR4 and CXCR7/RDC1. BMC Dev Biol 2007; 7: 23.

13 Naumann U, Cameroni E, Pruenster M, et al. CXCR7 functions as a scavenger for CXCL12 and CXCL11. PLoS One 2010; 5: e9175.

14 Levoye A, Balabanian $\mathrm{K}$, Baleux F, et al. CXCR7 heterodimerizes with CXCR4 and regulates CXCL12-mediated G protein signaling. Blood 2009; 113: 6085-6093.

15 Sierro F, Biben C, Martinez-Munoz L, et al. Disrupted cardiac development but normal hematopoiesis in mice deficient in the second CXCL12/SDF-1 receptor, CXCR7. Proc Natl Acad Sci USA 2007; 104: 14759-14764.

16 Luker KE, Gupta M, Steele JM, et al. Imaging ligand-dependent activation of CXCR7. Neoplasia 2009; 11: 1022-1035.
17 Zabel BA, Wang Y, Lewen S, et al. Elucidation of CXCR7-mediated signaling events and inhibition of CXCR4-mediated tumor cell transendothelial migration by CXCR7 ligands. J Immunol 2009; 183: 3204-3211.

18 Rajagopal S, Kim J, Ahn S, et al. $\beta$-Arrestin- but not G proteinmediated signaling by the "decoy" receptor CXCR7. Proc Natl Acad Sci USA 2010; 107: 628-632.

19 Gambaryan N, Perros F, Montani D, et al. Targeting of c-kit+ hematopoietic progenitor cells prevents hypoxic pulmonary hypertension. Eur Respir J 2011; 37: 1392-1399.

20 Hoshikawa Y, Nana-Sinkam P, Moore MD, et al. Hypoxia induces different genes in the lungs of rats compared with mice. Physiol Genomics 2003; 12: 209-219.

21 Miao Z, Luker KE, Summers BC, et al. CXCR7 (RDC1) promotes breast and lung tumor growth in vivo and is expressed on tumorassociated vasculature. Proc Natl Acad Sci USA 2007; 104: 15735-15740.

22 Neusser MA, Kraus AK, Regele H, et al. The chemokine receptor CXCR7 is expressed on lymphatic endothelial cells during renal allograft rejection. Kidney Int 2010; 77: 801-808.

23 Balabanian K, Lagane B, Infantino S, et al. The chemokine SDF-1/ CXCL12 binds to and signals through the orphan receptor RDC1 in T lymphocytes. J Biol Chem 2005; 280: 35760-35766.

24 Montani D, Perros F, Gambaryan N, et al. C-kit-positive cells accumulate in remodeled vessels of idiopathic pulmonary arterial hypertension. Am J Respir Crit Care Med 2011; 184: 116-123.

25 Mehta NN, Li M, William D, et al. The novel atherosclerosis locus at 10q11 regulates plasma CXCL12 levels. Eur Heart J 2011; 32: 963-971.

26 Bleul CC, Fuhlbrigge RC, Casasnovas JM, et al. A highly efficacious lymphocyte chemoattractant, stromal cell-derived factor 1 (SDF-1). J Exp Med 1996; 184: 1101-1109.

27 Burke DL, Frid MG, Kunrath CL, et al. Sustained hypoxia promotes the development of a pulmonary artery-specific chronic inflammatory microenvironment. Am J Physiol Lung Cell Mol Physiol 2009; 297: L238-L250.

28 Tuder RM, Groves B, Badesch DB, et al. Exuberant endothelial cell growth and elements of inflammation are present in plexiform lesions of pulmonary hypertension. Am J Pathol 1994; 144: 275-285.

29 Young KC, Torres E, Hatzistergos KE, et al. Inhibition of the SDF-1/CXCR4 axis attenuates neonatal hypoxia-induced pulmonary hypertension. Circ Res 2009; 104: 1293-1301.

30 Satoh K, Fukumoto Y, Nakano M, et al. Statin ameliorates hypoxiainduced pulmonary hypertension associated with down-regulated stromal cell-derived factor-1. Cardiovasc Res 2009; 81: 226-234.

31 Mazzinghi B, Ronconi E, Lazzeri E, et al. Essential but differential role for CXCR4 and CXCR7 in the therapeutic homing of human renal progenitor cells. J Exp Med 2008; 205: 479-490.

32 Raggo C, Ruhl R, McAllister S, et al. Novel cellular genes essential for transformation of endothelial cells by Kaposi's sarcomaassociated herpesvirus. Cancer Res 2005; 65: 5084-5095.

33 Yamaoka T, Frey MR, Dise RS, et al. . Specific epidermal growth factor receptor autophosphorylation sites promote mouse colon epithelial cell chemotaxis and restitution. Am J Physiol Gastrointest Liver Physiol 2011; 301: G368-G376. 